\title{
Package Type Not Stated
}

National Cancer Institute

\section{Source}

National Cancer Institute. Package Type Not Stated. NCI Thesaurus. Code C48626.

The package type is not stated or is unavailable. 